\title{
Relaxation towards phase-locked dynamics in long Josephson junctions
}

\author{
Salerno, M.; Grønbech-Jensen, Niels; Samuelsen, Mogens Rugholm
}

Published in:

Physical Review B

Link to article, DOI:

10.1103/PhysRevB.51.15613

Publication date:

1995

Document Version

Publisher's PDF, also known as Version of record

Link back to DTU Orbit

Citation $(A P A)$ :

Salerno, M., Grønbech-Jensen, N., \& Samuelsen, M. R. (1995). Relaxation towards phase-locked dynamics in long Josephson junctions. Physical Review B, 51(21), 15613-15616.

https://doi.org/10.1103/PhysRevB.51.15613

\section{General rights}

Copyright and moral rights for the publications made accessible in the public portal are retained by the authors and/or other copyright owners and it is a condition of accessing publications that users recognise and abide by the legal requirements associated with these rights.

- Users may download and print one copy of any publication from the public portal for the purpose of private study or research.

- You may not further distribute the material or use it for any profit-making activity or commercial gain

- You may freely distribute the URL identifying the publication in the public portal 


\title{
Relaxation towards phase-locked dynamics in long Josephson junctions
}

\author{
M. Salerno \\ Department of Theoretical Physics, University of Salerno, I84100 Salerno, Italy \\ N. Grønbech-Jensen \\ Theoretical Division, Los Alamos National Laboratory, Los Alamos, New Mexico 87545 \\ M. R. Samuelsen \\ Physics Department, The Technical University of Denmark, DK2800 Lyngby, Denmark
}

(Received 24 June 1994; revised manuscript received 6 February 1995)

\begin{abstract}
We study the relaxation phenomenon towards phase-locked dynamics in long Josephson junctions. In particular the dependence of the relaxation frequency for the equal time of flight solution on the junction parameters is derived. The analysis is based on a phase-locked map and is compared with direct numerical experiments performed both on the map and on the perturbed sine-Gordon equation. As an interesting result we find that very close to a bifurcation the relaxation frequency is exactly equal to the half of the step frequency, i.e., the frequency characterizing the period-one solution.
\end{abstract}

\section{INTRODUCTION}

Phase locking of fluxons in long Josephson junctions is a phenomenon which has attracted the attention of many researchers during the past years. ${ }^{1-4}$ From an experimental point of view, it gives the possibility of synchronizing arrays of Josephson oscillators at very high frequencies (of the order of hundreds $\mathrm{GHz}$ ) with an increase of the power output with respect to the corresponding one from a single junction and with a narrowing of the linewidth in the emitted radiation from the device. These features are quite attractive for practical applications of arrays of Josephson junctions as high-frequency oscillators in integrated superconductive receivers in radioastronomy and space communications. ${ }^{5}$ For a single Josephson junction, phase locking of fluxons to external microwave fields is understood as a boundary effect in the perturbed sineGordon equation. ${ }^{2,4,6}$ Although the phenomenon involves a partial differential equation (PDE), i.e., a system with infinite degrees of freedom, the existence of a "robust" localized object (fluxon) in the sine-Gordon equation reduces the problem to a two-dimensional map for the time of flight and for the energy of the fluxon inside the junction. ${ }^{2,7}$ In this approach, phase-locked dynamics in the PDE correspond to fixed points of the map, and the model has been shown to capture all the known experimental features of the phenomenon.

The aim of this paper is to investigate the effects of small disturbances on phase-locked dynamics of a single fluxon inside the junction. More precisely, we compute both analytically and numerically, the relaxation frequency towards the equal time of flight fixed points as a function of the bias current and of the other characteristic parameters of the junction. The analysis is performed in terms of the map model and the results are compared with numerical experiments performed both on the map and on the PDE. As a result we find that on fundamental steps the relaxation frequency towards the equal time of flight fixed point has its maximum in the middle of the step and goes to zero at the edges of the step. On subharmonic steps, however, the maximum relaxation frequency is reached for values of the dc bias close to the bifurcation points, and it is usually larger than the corresponding one observed on fundamental steps. Furthermore, we find that very close to a bifurcation the relaxation frequency is exactly equal to half of the step frequency, i.e., the frequency characterizing the periodone solution. This fact gives a natural explanation of the high sensitivity to the half-harmonic signal of the motion close to the bifurcation, thus leading to the stabilization of the fixed point against bifurcation as reported in previous papers. ${ }^{8-10}$ The results of our analysis are found in good agreement with direct numerical integrations on the PDE system. Although relaxation phenomena are usually not observed in real Josephson oscillators, we think there could be situations in which they could easily appear, such as, for example, when the fluxon is constantly disturbed (by, e.g., thermal noise, impurities, etc.) from its phase-locked motion.

The paper is organized as follows. In Sec. II we derive the analytical expression for the relaxation frequency in terms of the map model while in Sec. III we compare the analytical results with direct numerical experiments performed both on the map and on the full PDE system.

\section{ANALYSIS}

Let us start by recalling that the dynamics of a fluxon inside a long Josephson junction can be modeled by a two-dimensional dissipative map (for details see Ref. 2), which in the case of in-line geometry is written as

$$
\begin{aligned}
& T_{n+1}=T_{n}+\frac{1}{\alpha} \ln \left(\frac{\sqrt{U_{n}^{2}-1}}{c \sqrt{U_{n}^{2}-1}-s U_{n}}\right), \\
& U_{n+1}=c U_{n}-s \sqrt{U_{n}^{2}-1}+\frac{\pi}{2}\left[k_{\mathrm{dc}}+k_{\mathrm{rf}}\left(\omega_{\mathrm{rf}} T_{n+1}+\varphi\right)\right] .
\end{aligned}
$$


Here $k_{\mathrm{dc}}$ denotes the dc bias current, $\alpha$ is a dissipative parameter, $k_{\mathrm{rf}}$ is the amplitudes of the external field, and $\varphi$ is the phase. The constants $c, s$ in Eq. (1) are related to the length of the junction $L$ and to the dissipation by the relations $c=\cosh (\alpha L), s=\sinh (\alpha L)$. Phase-locked dynamics correspond to fixed points of the map with $T=$ $T_{n+1}-T_{n}$ denoting the time of flight of the fluxon inside the junction and $U_{n}$ being related to its energy. By fixing the locking condition $T=\frac{p 2 \pi}{q \omega}, p, q \in N$ one find the equal time of flight fixed points of $(1)$ as

$$
\begin{gathered}
U^{*}=\frac{c-\beta}{\sqrt{(c-\beta)^{2}-s^{2}}}, \\
T^{*}=\frac{1}{\omega_{\mathrm{rf}}}\left[\arcsin \left(\frac{\bar{k}-k_{\mathrm{dc}}}{k_{\mathrm{rf}}}\right)-\varphi\right]
\end{gathered}
$$

with $\beta=\exp \left(-\frac{\alpha m \pi}{\omega_{\mathrm{rf}}}\right)$ and $\bar{k}$ denoting the value of the bias current at which the rf-induced step crosses the unperturbed (i.e., with $k_{\mathrm{rf}}=0$ ) $I-V$ curve

$$
\bar{k}=\frac{2}{\pi}\left[U^{*}(1-c)+s \sqrt{U^{*^{2}}-1}\right] .
$$

To study relaxation dynamics towards fixed points we consider the linearized map at the fixed points, i.e.,

$$
\partial F=\left(\begin{array}{cc}
1 & a \\
b & \beta+a b
\end{array}\right)
$$

with

$$
a=\frac{-s\left(U^{*^{2}}-1\right)^{-3 / 2}}{\alpha \beta}, \quad b=\frac{\pi}{2} \omega_{\mathrm{rf}} k_{\mathrm{rf}} \cos \left(\omega_{\mathrm{rf}} T^{*}+\varphi\right) .
$$

A small initial disturbance $(\xi, \eta)$ will then evolve according to the linear system

$$
\left(\begin{array}{c}
\xi_{n+1} \\
\eta_{n+1}
\end{array}\right)=\partial F\left(\begin{array}{c}
\xi_{n} \\
\eta_{n}
\end{array}\right)
$$

Since the evolution of the $\xi$ variable depends on the value of the $\eta$ variable at previous time and vice versa, we can decouple system (5) by iterating it twice and then eliminating $\eta$ in favor of $\xi$, thus giving

$$
\begin{aligned}
& \xi_{n+2}=(1+a b+\beta) \xi_{n+1}-\beta \xi_{n}, \\
& \eta_{n+2}=(1+a b+\beta) \eta_{n+1}-\beta \eta_{n} .
\end{aligned}
$$

The solutions of these uncoupled second-order difference equations are easily obtained by introducing the shift operator $\Delta: \Delta \mu_{n}=\mu_{n+1}$, and rewriting them in the form

$$
\left(\Delta^{2}-\gamma \Delta+\beta\right) \mu_{n}=0,
$$

where $\gamma=1+a b+\beta$ and $\mu$ denotes both $\xi$ and $\eta$. The general solution of Eq. (7) is then obtained as

$$
\mu_{n}=A_{1} \lambda_{1}^{n}+A_{2} \lambda_{2}^{n}
$$

where $A_{i}, i=1,2$ are constants of integration to be fixed from the initial conditions and $\lambda_{1}, \lambda_{2}$, are solutions of the characteristic equation associated with (7), i.e.,

$$
\lambda^{2}+\gamma \lambda+\beta=0 .
$$

Note that Eq. (9) is just the secular equation of the Jacobian matrix (4), i.e., the $\lambda$ 's coincide with the eigenvalues of $\partial F$. By denoting $\xi_{0}, \eta_{0}$, the initial disturbance to the equal time of flight fixed points, and denoting with $\sigma_{i}, \delta_{i}$, the integration constants in Eq. (8) for, respectively, the variables $\xi, \eta$ we have that

$$
\begin{gathered}
\sigma_{i+1}=(-1)^{i} \frac{a \eta_{0}+\left(1-\lambda_{2-i}\right) \xi_{0}}{\lambda_{1}-\lambda_{2}} \\
\delta_{i+1}=(-1)^{i} \frac{b \xi_{0}+\left(\beta+a b-\lambda_{2-i}\right) \eta_{0}}{\lambda_{1}-\lambda_{2}}
\end{gathered} i=0,1 .
$$

From Eqs. $(8,10)$ the relaxation dynamics is finally obtained as

$$
\begin{gathered}
\xi_{n+1}=\left(\xi_{0}+a \eta_{0}\right)\left(\frac{\lambda_{1}^{n}-\lambda_{2}^{n}}{\lambda_{1}-\lambda_{2}}\right)-\xi_{0} \beta\left(\frac{\lambda_{1}^{n-1}-\lambda_{2}^{n-1}}{\lambda_{1}-\lambda_{2}}\right) \\
\eta_{n+1}=\left((\beta+a b) \eta_{0}+b \xi_{0}\right)\left(\frac{\lambda_{1}^{n}-\lambda_{2}^{n}}{\lambda_{1}-\lambda_{2}}\right)-\eta_{0} \beta\left(\frac{\lambda_{1}^{n-1}-\lambda_{2}^{n-1}}{\lambda_{1}-\lambda_{2}}\right) .
\end{gathered}
$$

From linear stability analysis it is known that the equal time of flight fixed points $\left(U^{*}, T^{*}\right)$ are stable if the following condition is satisfied: ${ }^{2,7}$

$$
-\Lambda \leq \eta_{1} \sin \left(\omega T^{*}\right) \leq 0
$$

with $\Lambda$ a constant given by

$$
\Lambda=\frac{4 \alpha \beta(1+\beta) s^{2}}{\pi \omega \sqrt{\left(1-2 \beta c+\beta^{2}\right)^{3}}} .
$$

The stability of the equal time of flight fixed point means that the eigenvalues of the Jacobian matrix are inside the unit circle and, for real eigenvalues, Eq. (11) implies an overdamped dynamics towards the fixed point. On the other hand, complex eigenvalues lie on the circle of radius $\sqrt{\beta}$ in the complex plane,${ }^{11,12}$ and they are complex conjugates of each other,

$$
\lambda_{1}=\lambda_{2}^{*}=\sqrt{\beta} e^{i \theta} .
$$

By substituting these expressions in Eq. (8) we find

$$
\begin{gathered}
\xi_{n+1}=\frac{\left(\xi_{0}+a \eta_{0}\right) \sqrt{\beta^{n-1}}}{\sin (\theta)} \sin (n \theta)-\frac{\xi_{0} \sqrt{\beta^{n}}}{\sin (\theta)} \sin [(n-1) \theta] \\
\eta_{n+1}=\frac{\left[(\beta+a b) \eta_{0}+b \xi_{0}\right] \sqrt{\beta^{n-1}}}{\sin (\theta)} \sin (n \theta) \\
-\frac{\eta_{0} \sqrt{\beta^{n}}}{\sin (\theta)} \sin [(n-1) \theta] .
\end{gathered}
$$

That is, the motion is a damped oscillation with frequency (in time unit given by $n$ ) which is just the argument $\theta$ of the complex eigenvalues

$$
\theta=\arctan \left(\frac{\sqrt{4 \beta-(1+a b+\beta)^{2}}}{1+a b+\beta}\right) .
$$

To convert this frequency in physical units we note that for the equal time of flight solution $n$ iterations of the map correspond to increase the time by $\left(\frac{p}{q} \frac{2 \pi}{\omega_{\mathrm{rf}}}\right) n$ so that the relaxation frequency $\Omega$ is given by

$$
\Omega=\frac{p}{q} \frac{\omega_{\mathrm{rf}}}{2 \pi} \theta
$$

In the next section we will compare these predictions with direct numerical iterations both of the map and of the PDE. 


\section{NUMERICAL ANALYSIS}

To check the results of the previous section let us first consider the relaxation dynamics on fundamental $\mathrm{rf}$ steps (i.e., steps on which the period-one motion has the same frequency as the external driver). On such steps the stability condition (12) is always satisfied for typical parameters of the junction. Furthermore the eigenvalues of the Jacobian matrix are always complex (except for values of the dc bias close to the edges of the step, where they become real), with $\theta$ reaching its maximum at $k_{\mathrm{dc}}=\bar{k}$, i.e., in the middle of the rf-induced step. This implies that on fundamental steps the relaxation frequency has its maximum in the central portion of the step and goes to zero at the edges. In Fig. 1 we report the $I-V$ curves obtained by iterating both the map and the PDE for parameter values $L=12, \alpha=0.15, \omega_{\mathrm{rf}}=0.35, k_{\mathrm{rf}}=0.05$, which represent the fundamental $(p=q=1)$ step (the averaged voltage is computed as $\langle\mathrm{V}\rangle=2 \pi / T)$. To distinguish the two curves in Fig. 1 an offset of 0.1 along the $x$ axis has been included. Note that the $I-V$ characteristic obtained by iterating the PDE is slightly shifted upward in current with respect to the curve obtained from the map model. This is a consequence of the fact that in the PDE there are losses which are not included in the map such as, for example, the losses during fluxon's reflections. In Fig. 2 the dynamics of the eigenvalues (stars) of the Jacobian matrix (4) as a function of the bias current (the dotted circle is the reference circle of radius $\sqrt{\beta}$ ) is reported. We see that by increasing the dc bias from the bottom to the top of the step the eigenvalues jump from the real axis (at the bottom) on the complex circle of radius $\sqrt{\beta}$, slowly move on it until the phase at $k_{\mathrm{dc}}=\bar{k}$ is reached, and then invert the motion to return on the real axis at the top of the step. In Fig. 3 we have reported the behavior of the relaxation frequency as a function of the bias current. In this figure the full curve is obtained from Eq. (14), the crosses represent experimental points obtained by iterating the map, while the squares are experimental points obtained from PDE calculations (the

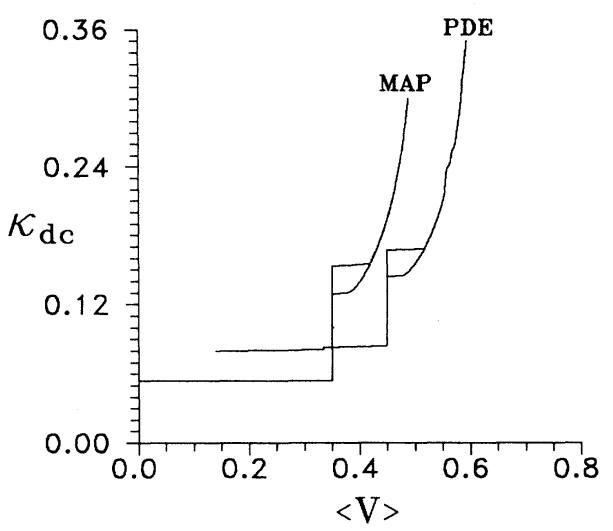

FIG. 1. $I-V$ characteristics obtained from the map and the PDE for parameter values $L=12, \alpha=0.015, \omega_{\text {rf }}=0.35$, $k_{\mathrm{rf}}=0.05$. To avoid overlapping the PDE curve is shifted by 0.1 in voltage.

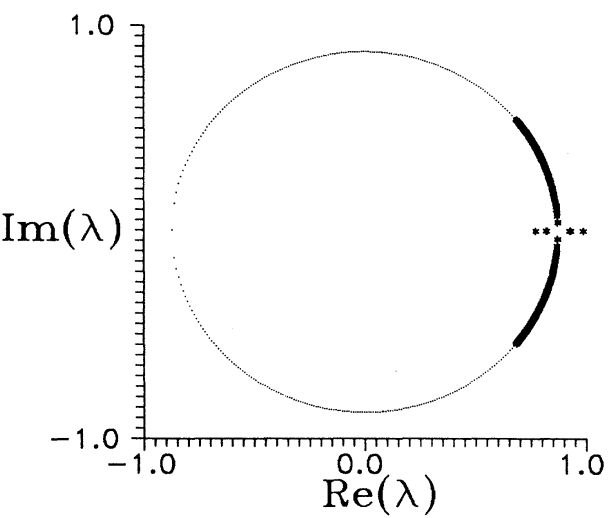

FIG. 2. Dynamics of the eigenvalues of Eq. (4) as $k_{\mathrm{dc}}$ is varied along the step of Fig 1.

PDE results are shifted a bit upward in current with respect to the map results for the same reason as before). We see that the relaxation dynamics is overdamped (real eigenvalues) at the edges of the step and damped in the central portion as predicted by our analysis. We also remark that the maximum frequency in this case is lower than the maximum allowed frequency (which is obtained for $\theta=\pi$ ). This situation is typical of fundamental steps on which the equal time of flight solution is stable for all values of bias along the step. For subharmonic steps, however, one sees from Eq. (12) that the range of stability is more narrow and one of the eigenvalues can leave the unit circle at -1 . As shown in Refs. 11 and 12 this happens after the eigenvalues have made a full excursion on the circle of radius $\sqrt{\beta}$ in the complex plane, i.e., after the phase has increased from zero (for $k_{\mathrm{dc}}$ close to the bottom) to $\pi$ (close to the bifurcation). Due to the symmetry with respect to $\bar{k}$ the same situation holds true also in the upper portion of the step. From Eq. (14) we expect then that in proximity of the bifurcation the relaxation frequency reaches its maximum (which is just

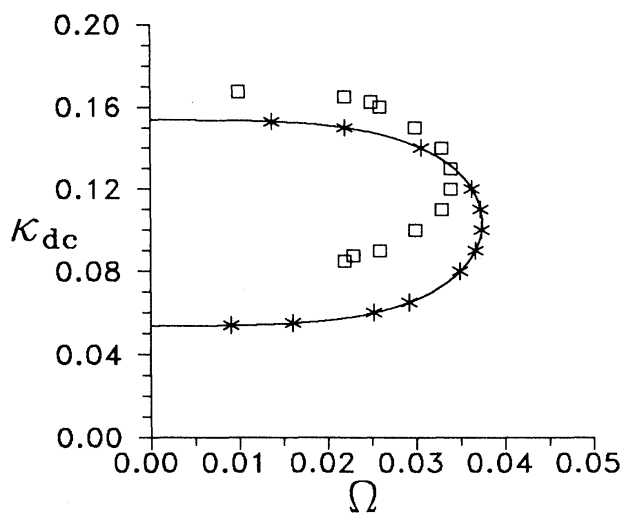

FIG. 3. Dependence of the relaxation frequency on the bias current for the step of Fig. 1. The continuous curve is obtained from Eq. (14), the stars are experimental points obtained by iterating the map, the squares are experimental points from PDE calculations. 


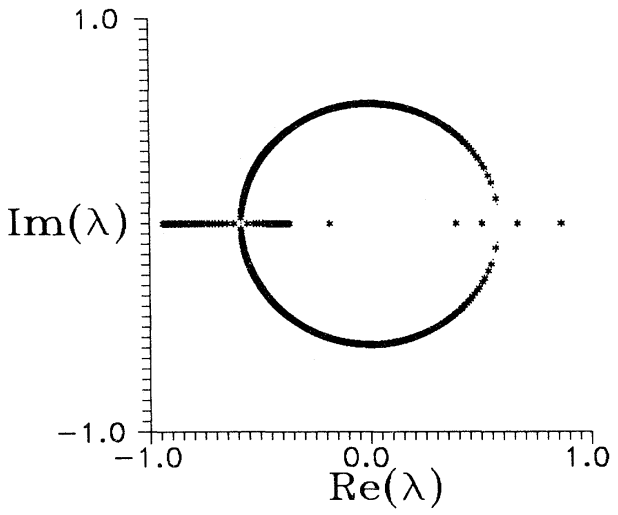

FIG. 4. Same as Fig. 2 but for parameter values $L=12$, $\alpha=0.03, \omega_{\mathrm{rf}}=0.35, k_{\mathrm{rf}}=0.035$.

half the step frequency $\left.\frac{p}{q} \omega_{\text {rf }}\right)$, while it is zero at the edges of the step. To check this, we consider the bifurcation of the equal time of flight solution into a period-two solution observed, both in the map and in the PDE, on the $p=1, q=2$ step, for parameter values $L=12, \alpha=0.03$, $\omega_{\mathrm{rf}}=0.35$, and $k_{\mathrm{rf}}=0.035$. In Fig. 4 we report the motion of the eigenvalues of Eq. (4) in correspondence with this bifurcation. We see that the eigenvalues move around the circle going from $\sqrt{\beta}$ (for $k_{\mathrm{dc}}$ close to the edge of the step) to $-\sqrt{\beta}$ (for $k_{\mathrm{dc}}$ close to bifurcation) so that the relaxation frequency has its maximum in proximity of the bifurcation point. This implies that the motion is overdamped (real eigenvalues) at the bottom while it is a damped oscillation with frequency $\Omega=0.0875$, close to the bifurcation point. This is readily seen in Fig. 5 in which the relaxation frequencies derived both from the map and the PDE are plotted as a function of the bias current along the step. In this figure the full curves are obtained from expression (14), the stars are obtained by direct iterations of the map (the splitting in two branches is due to the existence of two equal time of flight solutions), and the squares denote experimental points from PDE numerical calculations. We see here that the PDE and map results are shifted more than in Fig. 3 as a con-

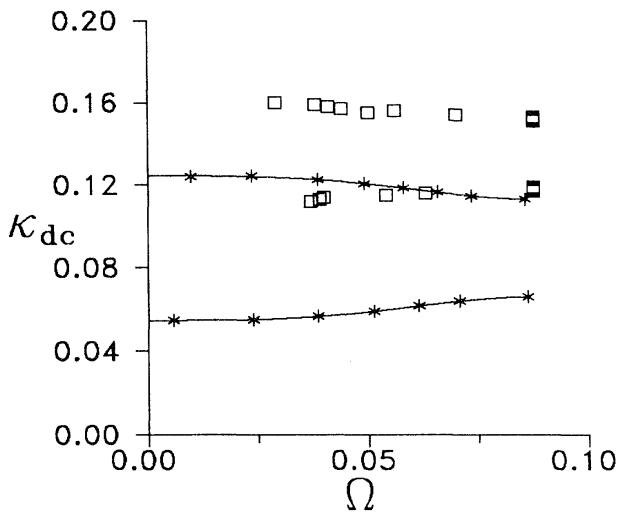

FIG. 5. Same as Fig. 3 but for parameters as in Fig. 4.

sequence of the higher dissipations on subharmonic steps in the PDE calculations. It is remarkable that in both the map and the PDE, just before the bifurcation, the relaxation frequency is equal to half the step frequency, i.e., the frequency characterizing the period-one solution. It is also interesting that close to the bifurcation point, the frequency becomes constant at its maximum value. This is of course where the eigenvalues hit the negative real axis. This gives a natural explanation of the sensitivity and effectiveness of external half-harmonic signals, in stabilizing the fixed point against bifurcation. ${ }^{8-10} \mathrm{We}$ finally note that there is a good agreement between the analytical previsions and the experimental results both in the map and in the PDE, thus confirming the validity of the map model and the low-dimensionality nature of the phenomenon in the infinite-dimensional system.

\section{ACKNOWLEDGMENTS}

Parts of this work were performed under the auspices of the U.S. DOE. Financial support from the INFM (Istituto Nazionale di Fisica della Materia) sezione di Salerno is also acknowledged.
${ }^{1}$ M. Scheuermann, J. T. Chen, and J. J. Chen, J. Appl. Phys. 54, 3286 (1984).

${ }^{2}$ M. Salerno, M. R. Samuelsen, G. Filatrella, S. Pagano, and R. D. Parmentier, Phys. Rev. B 41, 6641 (1990).

${ }^{3}$ B. A. Malomed, Phys. Rev. B 41, 1037 (1990).

${ }^{4}$ N. Grønbech-Jensen, Phys. Rev. B 47, 5504 (1993).

${ }^{5}$ R. Monaco, S. Pagano, and G. Costabile, Phys. Lett. A 131, 122 (1988).

${ }^{6}$ M. Salerno and M. R. Samuelsen, Phys. Lett. A 156, 293 (1991).
${ }^{7}$ M. Salerno, Phys. Lett. A 144, 453 (1990).

${ }^{8} \mathrm{~K}$. Wiesenfeld and M. McNamara, Phys. Lett. 55, 13 (1985).

${ }^{9}$ H. Svensmark and M. R. Samuelsen, Phys. Rev. B 41, 4181 (1990).

${ }^{10}$ M. Salerno, Phys. Rev. B 44, 2720 (1991).

${ }^{11}$ M. Salerno, Phys. Lett. A 160, 419 (1991).

${ }^{12}$ N. Grønbech-Jensen, M. Salerno, M. R. Samuelsen, Phys. Rev. B 46, 308 (1992). 